\title{
Inhibition of miR-34a ameliorates cerebral ischemia/reperfusion injury by targeting brain-derived neurotrophic factor
}

\section{Type}

Research paper

\section{Keywords}

ischemia/reperfusion injury, BDNF, miR-34a, oxidative stress, neuronal apoptosis

\begin{abstract}
Introduction

Oxidative stress and neuronal apoptosis are strongly associated with the pathogenesis of ischemic stroke. In this study, we aimed to determine whether miR-34a was involved in ischemia/reperfusion $(\mathrm{I} / \mathrm{R})$ injury, oxidative stress, and neuronal apoptosis by targeting brain-derived neurotrophic factor (BDNF).

Material and methods

Rats received middle cerebral artery occlusion (MCAO) surgery to simulate I/R injury. At $24 \mathrm{~h}$ after MCAO surgery, neurological deficits and infarct volumes were evaluated according to Longa's scale and 2,3,5-triphenyltetrazolium (TTC) chloride staining. Neuronal apoptosis was assessed by terminal deoxynucleotidyl transferase-mediated dUTP nick-end labeling (TUNEL), and the expression of miR-34a and associated proteins were detected by quantitative reverse-transcription polymerase chain reaction (qRT-PCR), and western blotting. Several markers of oxidative stress were detected using commercial kits, and the interaction between miR-34a and BDNF was measured by RNA immunoprecipitation (RIP).
\end{abstract}

\section{Results}

The results showed that miR-34a was upregulated $(p<0.05)$, whereas BDNF was downregulated ( $p$ $<0.05$ ) in the MCAO rats, and this negative correlation was accompanied by clear oxidative stress and neuronal apoptosis. RIP demonstrated a clear interaction between miR-34a and BDNF. Furthermore, miR-34a was also found to inhibit oxidative stress and neuronal apoptosis, increase BDNF expression, and ameliorate neurological deficits and infarct volumes $(p<0.05)$ seen in the MCAO rats.

\section{Conclusions}

These data suggested that inhibition of miR-34a ameliorated cerebral ischemia/reperfusion injury by targeting BDNF. This mechanism represents a novel and promising target for the treatment of strokes. 
1 Inhibition of miR-34a ameliorates cerebral ischemia/reperfusion injury by

2 targeting brain-derived neurotrophic factor

3 Running title: miR-34a targets BDNF in strokes

4 Shilin Zhu ${ }^{1, \#, ~ J i a n g h o n g ~ T a n g ~}{ }^{2, \#}$, Lan Lan ${ }^{3}$, Feng Su ${ }^{4}$

$5{ }^{1}$ Department of Nursing, The Second Affiliated Hospital of Hunan University of

6 Chinese Medicine, Changsha, Hunan 410005, China.

$7 \quad{ }^{2}$ Department of Gerontology, The Second Affiliated Hospital of Hunan University of

8 Chinese Medicine, Changsha, Hunan 410005, China.

$9{ }^{3}$ Department of Orthopedics, The Second Affiliated Hospital of Hunan University of

10 Chinese Medicine, Changsha, Hunan 410005, China.

$11{ }^{4}$ Department of Emergency, Xiangya Hospital, Central South University, Changsha,

12 Changsha, Hunan 410008, China.

13 Correspondence to: $D r$ Feng Su, Department of Emergency, Xiangya Hospital, Central

14 South University, 87 Xiang Ya Road, Kaifu, Changsha, Hunan 410008, P.R. China

15 Tel: +8613908466487

16 Email: sufengdr@163.com 
$17 \quad{ }^{\#}$ Contributed equally

18 Highlights: 1. The putative interaction between miR-34a and BDNF was established by 19 RIP.

20 2. Inhibition of miR-34a ameliorated I/R injury by targeting BDNF.

213 . Inhibition of miR-34a alleviated oxidative stress and neuronal apoptosis.

22 


\section{Abstract}

24 Introduction: Oxidative stress and neuronal apoptosis are strongly associated with the

25 pathogenesis of ischemic stroke. In this study, we aimed to determine whether miR-34a

26 was involved in ischemia/reperfusion (I/R) injury, oxidative stress, and neuronal

27 apoptosis by targeting brain-derived neurotrophic factor (BDNF).

28 Material and methods: Rats received middle cerebral artery occlusion (MCAO)

29 surgery to simulate I/R injury. At $24 \mathrm{~h}$ after MCAO surgery, neurological deficits and

30 infarct volumes were evaluated according to Longa's scale and 2,3,5-

31 triphenyltetrazolium (TTC) chloride staining. Neuronal apoptosis was assessed by

32 terminal deoxynucleotidyl transferase-mediated dUTP nick-end labeling (TUNEL), and

33 the expression of miR-34a and associated proteins were detected by quantitative

34 reverse-transcription polymerase chain reaction (qRT-PCR), and western blotting.

35 Several markers of oxidative stress were detected using commercial kits, and the

36 interaction between miR-34a and BDNF was measured by RNA immunoprecipitation

37 (RIP).

38 Results: The results showed that miR-34a was upregulated $(p<0.05)$, whereas BDNF

39 was downregulated $(p<0.05)$ in the MCAO rats, and this negative correlation was

40 accompanied by clear oxidative stress and neuronal apoptosis. RIP demonstrated a clear 
41 interaction between miR-34a and BDNF. Furthermore, miR-34a was also found to

42 inhibit oxidative stress and neuronal apoptosis, increase BDNF expression, and

43 ameliorate neurological deficits and infarct volumes $(p<0.05)$ seen in the MCAO rats.

44 Conclusions: These data suggested that inhibition of miR-34a ameliorated cerebral

45 ischemia/reperfusion injury by targeting BDNF. This mechanism represents a novel and

46 promising target for the treatment of strokes.

47 Keywords: ischemia/reperfusion injury, miR-34a, BDNF, oxidative stress, neuronal

48 apoptosis

\section{Introduction}

50 Epidemiological evidence suggests that strokes represent the second largest cause of

51 death worldwide, and in approximately $87 \%$ of victims, the cause is ischemia (ischemic

52 stroke) due to thrombosis or embolism $[1,2]$. The factors associated with ischemic

53 strokes are principally divided into uncontrollable (such as old age, male, and ethnic

54 minorities, mainly Afro-Caribbean) and controllable factors (such as hypertension,

55 diabetes, and hypercholesterolemia). The controllable factors can be managed by

56 removing the blockages within blood vessels. However, when this is performed,

57 reperfusion and reoxygenation are also associated with damage [3] 
58 (ischemia/reperfusion (I/R) injury), which is manifested as oxidative stress, damage to

59 the blood-brain barrier (BBB), neurovascular dysfunction, and neuronal death, all

60 representing significant obstacles to therapy [4]. Thus, inhibition of the I/R injury may

61 represent a novel therapy and provide a better long-term prognosis for stroke patients.

62 Accumulating evidence has implicated oxidative stress in I/R injury [5], and a

63 sudden burst of oxidative stress following I/R can promote neuronal apoptosis [6].

64 Although the underlying mechanisms of oxidative stress-induced neuronal apoptosis

65 remain unknown, inhibition of oxidative stress in the early stages of a stroke may

66 represent a promising strategy for suppressing neuronal apoptosis and alleviating injury.

67 Recently, many researchers have found that brain-derived neurotrophic factor (BDNF),

68 a member of the neurotrophin family, regulates neuron survival, differentiation, and

69 apoptosis through different pathways, and is of great significance for maintaining the

70 development and function of the nerves [7, 8]. More importantly, BDNF is closely

71 associated with oxidative stress and neuronal apoptosis in cell and animal models of I/R

$72[9]$.

73 MicroRNAs (miRNAs) are crucial post-transcriptional regulators of gene

74 expression, and the expression of some, including miR-141, miR-429, miR-200, miR-

75 182, miR-183, miR-33, miR-125a-5p, miR-155, miR-34a, and miR-96, are altered after 
reperfusion [10]. Among these, miR-34a plays an important role in oxidative stress and

77 apoptosis in human mesenchymal stromal/stem cells, human umbilical vein endothelial

78 cells [11], and vascular endothelial cells [12]. Interestingly, miR-34a expression is

79 decreased in patients with stroke [13] and rats exposed to middle cerebral artery

80 occlusion (MCAO) [14]. Furthermore, in vitro evidence suggests that miR-34a triggers

81 the breakdown of the BBB and abnormal oxidative phosphorylation in endothelial cells

82 [15]. Moreover, previous studies have indicated that miR-34a is a potential target for

83 neuropathology [16]. However, whether miR-34a is implicated in oxidative stress-

84 induced neuronal apoptosis following I/R injury by targeting BDNF remains unknown.

85 In this study, we attempted to determine the relationship between miR-34a and

86 BDNF in the MCAO rat model. Furthermore, an miR-34a inhibitor was administered to

87 determine the potential role of miR-34a as a mediator of oxidative stress and oxidative

88 stress-induced neuronal apoptosis. RNA immunoprecipitation (RIP) was also performed

89 to establish putative interactions between miR-34a and BDNF.

90 Materials and Methods

$91 \quad$ Animals and study groups

92 A total of 60 male Sprague-Dawley rats $(250-300 \mathrm{~g})$ were obtained from the Animal 
93 Experiment Center at the Institute of Radiation Medicine of the Chinese Academy of

94 Medical Sciences. The rats were fed with standard chow, and drinking water was freely

95 available. Animals were kept in a 12:12 h light/dark cycle, a humidity of $40 \%$, and a

96 temperature of $22 \pm 2{ }^{\circ} \mathrm{C}$. Rats were randomly divided into the sham group $(n=15)$,

97 MCAO group $(n=15)$, miR-34a inhibitor negative control group (reflected by miR-34a

98 inhibitor NC in the figures; $n=15)$, and miR-34a inhibitor group $(n=15)$. All rats

99 except for those in the sham group underwent MCAO surgery. In addition, rats in the

100 negative control group and inhibitor group received treatment with a miR-34a inhibitor

101 negative control or a miR-34a inhibitor by intracerebroventricular injection (ICV

102 injection). $2 \%$ pentobarbital sodium $(30 \mathrm{mg} / \mathrm{kg}$ ) was used to induce euthanasia. The use

103 of experimental animals in the present study was carried out in accordance with the

104 Guide for the Care and Use of Laboratory Animals of the National Institutes of Health

105 (USA) [17]. All experimental protocols were approved by the Animal Ethics Committee

106 of the Institute of Radiation Medicine of the Chinese Academy of Medical Sciences.

107 The experiments were conducted at The Institute of Radiation Medicine of the Chinese

108 Academy of Medical Sciences.

109 MCAO surgery

110 Surgery was performed according to previous studies [18]. Briefly, the rats were 
111 anesthetized using $30 \mathrm{mg} / \mathrm{kg}$ pentobarbital sodium (2\% solution, intraperitoneal

112 injection). The right common carotid artery, internal carotid artery, and external carotid

113 artery were exposed, and the body temperatures were continuously monitored and

114 maintained at $36.5-37.5^{\circ} \mathrm{C}$ with a thermostatic blanket. Following $1 \mathrm{~h}$ of transient

115 occlusion, the cerebral blood flow was restored by removing the suture for $24 \mathrm{~h}$.

116 Subsequently, the right common carotid artery, external carotid artery, and internal

117 carotid artery were exposed via a midline cervical incision. A piece of $4 / 0$ monofilament

118 nylon suture with a heat-induced rounded tip was inserted through the right internal

119 carotid artery to the base of the middle cerebral artery, which occluded the blood flow to

120 the cortex and striatum. For the sham surgery, all arteries were exposed during the

121 surgical period, but the filament was not inserted into the MCA. Following surgery, the

122 rats were housed individually and closely monitored for changes in behavior and vital

123 signs. The MCAO model was considered successfully established when the following

124 observations were indicated: i) Horner syndrome occurred in the ipsilateral (left side)

125 when the rat displayed wakefulness after surgery; ii) the forelimbs did not completely

126 stretch; and iii) contralateral circling occurred when walking. Simultaneously, the Zea-

127 Longa neurological deficit scores were calculated. Scores of 2 and 3 were included in

128 the MCAO model. The neurological scores were blindly assessed independently by two

129 pretrained technicians when the rats awoke after MCAO surgery according to the Zea- 
130 Longa neurological deficit scores [19], The Zea-Longa assessment criteria were as

131 follows: Score 0, normal, no neurological sign; score 1, cannot completely stretch

132 contralateral forelimbs; score 2, contralateral circling when walking; score 3,

133 contralateral fall over when walking; and score 4, cannot walk and lowered

134 consciousness.

\section{Intracerebroventricular injection}

136 The miR-34a inhibitor negative control and miR-34a inhibitor were purchased from

137 RiboBio (Guangzhou, China). Five minutes after MCAO surgery, the rats in the miR-

13834 a negative control group were given miR-34a inhibitor negative control $(5 \mathrm{mg} / \mathrm{ml})$

139 according to the manufacturer's protocol (RiboBio, Guangzhou, China) and the rats in

140 the miR-34a inhibitor group were given miRNA-34a inhibitor $(5 \mathrm{mg} / \mathrm{ml})$. These were

141 administered by ICV injection through a skull hole into the left lateral cerebral

142 ventricles (coordinates: $0.9 \mathrm{~mm}$ caudal, $1.4 \mathrm{~mm}$ lateral, and $4.6 \mathrm{~mm}$ deep with respect to

143 Bregma) no more than 5 min after MCAO [20].

\section{Scoring of neurological deficits}

145 Scoring was performed $24 \mathrm{~h}$ after MCAO according to Longa's scale [21]. Briefly, this

146 scale was as follows: score 0 , no deficits; score 1 , difficulty in extending the 
147 contralateral forelimb; score 2, mild circling to the contralateral side; score 3, severe

148 circling to the contralateral side; and score 4, no spontaneous motor activity.

\section{Sample collection}

150 After scoring the neurological deficits for $24 \mathrm{~h}$, the animals were euthanatized with 30

$151 \mathrm{mg} / \mathrm{kg}$ pentobarbital sodium. The brains from a portion of the rats ( $n=5$ per group)

152 were rapidly removed, sliced into five coronal sections, and used for 2,3,5-

153 triphenyltetrazolium chloride (TTC) staining. A further portion of the rats $(n=5$ per

154 group) was sacrificed after their cortices had been collected under anesthesia. The

155 cortices were stored at $-80^{\circ} \mathrm{C}$ for quantitative reverse-transcription polymerase chain

156 reaction (qRT-PCR), western blot, RIP, and the detection of oxidative stress-related

157 markers. A portion of rats ( $n=5$ per group) received intracardial perfusion first with

158 saline and then with 4\% paraformaldehyde in PBS. The brains from these animals were

159 removed, and $10-\mu \mathrm{m}$ frozen sections were prepared for terminal deoxynucleotidyl

160 transferase-mediated dUTP nick-end labeling (TUNEL) staining.

161 Measurement of infarct area

162 Sections were incubated in 2\% TTC solution (Sigma-Aldrich, St. Louis, MO, USA) at

$16337^{\circ} \mathrm{C}$ for $30 \mathrm{~min}$ and incubated in fixative (4\% formaldehyde) for $24 \mathrm{~h}$. From these 
164 sections, images were captured using a digital camera, and Image-Pro Plus 6.0 software

165 was used to measure the infarct volume according to a previous study [22].

\section{RT-qPCR}

167 Hippocampus tissue from three rats from each group was taken for total RNA extraction

168 using the TRIzolRNAiso Plus kit (TaKaRa, Dalian, China). The Prime Script RT

169 reagent kit with gDNA Eraser (TaKaRa) was used for reverse transcription of total RNA

170 according to the manufacturer's instructions. Next, RT-qPCR was performed with

171 SYBR Premix Ex TaqII (TaKaRa) using a CFX96 detection system (Bio-Rad, Hercules,

172 CA, USA). The primers used were as follows [23]: miRNA-34a forward 5'-

173 CATGGCAGTGTCTTAGCTGGTT-3'; reverse 5'-CAGTGCAGGGTCCGAGGTAT-3',

174 and U6 forward 5'-CTCGCTTCGGCAGCACA-3'; reverse 5'-

175 AACGCTTCACGAATTTGCGT-3'. U6 snRNA was used to standardize the expression

176 levels of miRNA-34a.

177 Western blot

178 Hippocampal tissues from three rats in each group were taken and washed twice with

179 PBS. Then, they were lysed in lysis buffer (Boster, Wuhan, China), vortexed, and

180 centrifuged at $12,000 \times \mathrm{g}$ for $30 \mathrm{~min}$ at $4{ }^{\circ} \mathrm{C}$. The supernatant was removed, and the 
181 total protein concentration was measured using a BCA kit (Beyotime). Total protein

182 was separated using $10 \%$ sodium dodecyl sulfate-poly-acrylamide gel electrophoresis

183 (SDS-PAGE). Then, proteins were transferred to a polyvinylidene difluoride (PVDF)

184 membrane (Millipore Corporation, Billerica, MA, USA). After blocking with 5\%

185 skimmed milk in Tris-buffered saline/Tween-20 (TBST) for $1 \mathrm{~h}$ at room temperature,

186 the membranes were incubated with primary antibodies overnight at $4{ }^{\circ} \mathrm{C}$. The

187 following primary antibodies were used: rabbit anti-BDNF (1:1,000; Abcam,

188 Cambridge, MA, USA), rabbit anti-cleaved caspase-3 (1:1,000; Abcam), rabbit anti-Bax

189 (1:1,000; Abcam), rabbit anti-Bcl-2 (1:1,000; Abcam), and rabbit anti-GAPDH

190 (1:3,000; Abcam). After washing three times in TBST, the membranes were incubated

191 with horseradish peroxidase (HRP)-conjugated secondary antibody (1:3,000; Abcam)

192 for $1 \mathrm{~h}$ at room temperature. Finally, images were visualized using chemiluminescence

193 (Boster) and analyzed by Quantity One software (Bio-Rad, Hercules, CA, USA).

194 RIP

195 RIP analysis was performed to detect a possible interaction between miR-34a and

196 BDNF using the Magna RIP RNA-binding protein immunoprecipitation kit (Millipore

197 Corporation) in accordance with the manufacturer's instructions. Co-precipitated RNA

198 was detected by RT-qPCR. Here, the anti-IgG represented the negative control, and the 
199 input represented the cell lysates.

\section{Detection of oxidative stress-related markers}

201 The levels of ROS, glutathione (GSH), glutathione peroxidase (GSH-Px), and

202 glutathione reductase (GR) were detected using their appropriate kits (Beyotime,

203 Shanghai, China) and according to the manufacturer's instructions.

204 TUNEL staining

205 Brain tissues were taken from 15 rats from each group to detect the rate of apoptosis in

206 the CA1 area of the hippocampus. Paraffin-embedded sections from the hippocampus

207 were placed onto poly-lysine coated slides. Neuronal apoptosis was determined by a

208 one-step TUNEL apoptosis detection assay kit (Beyotime) according to the

209 manufacturer's instructions. Mouse anti-NeuN (1:100; Millipore Corporation) was used

210 to label neuronal nuclei, and DAPI (Beyotime) was used to counterstain the nuclei.

211 Images were captured using a fluorescence microscope (Olympus, Tokyo, Japan).

212 Neuronal apoptosis in the penumbral region was assessed by overlapping ratios between

213 NeuN and TUNEL.

\section{Statistical analysis}


215 Data were expressed as mean \pm standard deviation and analyzed by one-way analysis of

216 variance (ANOVA) followed by post hoc Tukey's test. Differences at the $p<0.05$ level

217 were deemed to be statistically significant.

218 
219 Results

220 Inhibition of miR-34a ameliorates ischemic infarction and neurological deficits.

221 To determine the effects of miR-34a on I/R injury, MCAO rats received a treatment with

222 the miR-34a inhibitor negative control or the miR-34a inhibitor. We observed that the

223 expression of miR-34a was increased in the MCAO group compared with the sham

224 group (Fig. 1D, $p<0.05$ ). There was no significant change in the expression of miR-34a

225 between the negative control group and the MCAO group (Fig. 1D, $p>0.05$ ). However,

226 compared with the MCAO group, the expression of miR-34a was consistently and

227 significantly decreased in the inhibitor group (Fig. 1D, $p<0.05$ ). Next, we determined

228 the infarct volumes and neurological deficit scores in these groups. Our results showed

229 that there was a larger infarct volume (Fig. 1A and 1B, $p<0.05$ ) and a more serious

230 neurological deficit score (Fig. 1C, $p<0.05$ ) in the MCAO group than the sham group.

231 However, these differences did not reach statistical significance between the negative

232 control group and the MCAO group (Fig. 1A and 1B, $p>0.05$, Fig. 1C, $p>0.05$ ).

233 Notably, however, we found a lower infarct volume (Fig. 1A and 1B, $p<0.05$ ) and

234 lower neurological deficit scores (Fig. 1C, $p<0.05$ ) in the miR-34a inhibitor group than

235 the MCAO group.

236 Inhibition of miR-34a upregulates BDNF expression. 
237 It has been reported that miR-34a can inhibit the expression of BDNF, as assessed by

238 luciferase reporter assays [24]. In agreement with this, we found a strong interaction

239 between miR-34a and BDNF using RIP analysis (Fig. 2A). In addition, western blotting

240 revealed that BDNF expression was decreased in the MCAO group compared with the

241 sham group (Fig. 2B and 2C, $p<0.05$ ). However, there was no statistically significant

242 difference in the expression of BDNF between the miR-34a negative control group and

243 the MCAO group (Fig. 2B and 2C, $p>0.05$ ). However, BDNF expression was

244 upregulated following the addition of the miR-34a inhibitor compared with the MCAO

245 group (Fig. 2B and 2C, $p<0.05)$.

246 Inhibition of miR-34a alleviates oxidative stress following MCAO.

247 To detect the effects of miR-34a on oxidative stress following MCAO, we monitored

248 the levels of oxidative stress markers, such as ROS, GSH, GSH-Px, and GR. We found

249 that ROS levels (Fig. 3A, $p<0.05$ ) were increased, but GSH (Fig. 3B, $p<0.05$ ), GSH-

250 Px (Fig. 3C, $p<0.05$ ), and GR (Fig. 3D, $p<0.05$ ) were decreased significantly in the

251 MCAO group compared with the sham group. However, there were no significant

252 differences in ROS, GSH, GSH-Px, or GR levels between the miR-34a negative control

253 group and MCAO group (Fig. 3A-3D, $p>0.05$ ). However, the miR-34a inhibitor group

254 displayed decreased ROS (Fig. 3A, $p<0.05$ ) and increased GSH (Fig. 3B, $p<0.05$ ), 
256 group.

257 Inhibition of miR-34a reduces neuronal apoptosis following MCAO.

258 It is well known that oxidative stress can induce neuronal apoptosis. Therefore, we

259 measured the extent of neuronal apoptosis by double-label immunofluorescence

260 staining, TUNEL, NeuN, and western blot. The MCAO group showed greater neuronal

261 apoptosis than the sham group (Fig. $4 \mathrm{~A}$ and $4 \mathrm{~B}, p<0.05$ ). While there was no

262 significant difference in neuronal apoptosis between the miR-34a negative control group

263 and MCAO group, decreased neuronal apoptosis was clearly seen in the miR-34a

264 inhibitor group compared with the MCAO group (Fig. 4A and 4B, $p<0.05$ ). In

265 addition, cleaved-caspase-3 and Bax expressions were increased, whereas Bcl-2

266 expression was decreased in the MCAO group compared with the sham group (Fig. 4C

267 and 4D, $p<0.05$ ). The addition of the miR-34a inhibitor reversed this trend (Fig. 4C

268 and $4 \mathrm{D}, p<0.05)$.

269 Discussion

270 It has been reported that after MCAO intervention, the level of ROS increases

271 significantly, which activates different signaling pathways to generate oxidative stress 
273 free radicals and increases the content of SOD and GSH-Px in neurons, thus reducing

274 the accumulation of free radicals [27], and protecting neurons after cerebral ischemia

275 [28]. In our present study, we have shown high expression levels of miR-34a and low expression of BDNF $48 \mathrm{~h}$ after MCAO, which was paralleled by clear oxidative stress and neuronal apoptosis. However, the inhibition of miR-34a could increase the expression of BDNF and suppress oxidative stress and neuronal apoptosis in MCAO rats, resulting in reduced ischemic infarction and neurological deficits. These results suggested that inhibition of miR-34a expression could ameliorate the injury caused by I/R through the inhibition of BDNF expression. Therefore, miR-34a may be a novel and promising target for the suppression of injuries associated with I/R.

283 During the ischemic phase of a stroke, interruption of blood flow causes a severe reduction in oxygen and nutrients at the ischemic area. This causes abnormal oxidative phosphorylation and the accumulation of metabolites, which cause an imbalance between oxidative stress and antioxidant mechanisms [29]. At reperfusion however,

287 sudden blood flow is restored causing an acute increase in ROS to the ischemic area, 288 representing the most important trigger for oxidative stress [5]. Next, anabatic oxidative 289 stress induces neuronal injuries, including apoptosis [30]. In this study, we found that 290 increased ROS but decreased GSH, GSH-Px, and GR were accompanied by obvious 
291 neuronal apoptosis $48 \mathrm{~h}$ after MCAO. Oxidative stress and oxidative stress-induced

292 neuronal apoptosis are closely related to the pathophysiology of strokes [31, 32], and

293 many studies suggest that miRNAs have a regulatory role in oxidative stress. For

294 example, miR-23a-3p can increase the production of manganese SOD and decrease the

295 production of peroxidative nitric oxide and 3-nitrotyrosine in MCAO mice and $\mathrm{H}_{2} \mathrm{O}_{2}$ -

296 treated neuro-2a cells, resulting in the downregulation of cleaved caspase-3 [33]. In

297 addition, the inhibition of miR-106b-5p downregulates the malondialdehyde content

298 and Bax expression but upregulates Bcl-2 expression and SOD activity in MCAO rats

299 and glutamate-treated PC12 cells, thereby inhibiting oxidative damage and neuronal

300 apoptosis [34]. In this study, we found that oxidative stress and neuronal apoptosis were

301 positively related to high levels of miR-34a expression in the MCAO rats. Moreover,

302 oxidative stress, neuronal apoptosis, ischemic infarction, and neurological deficits were

303 dramatically decreased in MCAO rats when an miR-34a inhibitor was used. These data

304 indicated that miR-34a was involved in oxidative stress and neuronal apoptosis, which

305 is consistent with previous studies $[11,35,36]$.

306 As early as 1993, Mattson et al. found that neurotrophic factors contribute to

307 calcium homeostasis and the suppression of ROS production [37]. Since then, research

308 has confirmed a role for neurotrophic factors in oxidative stress and oxidative stress-

309 associated cell injuries, such as apoptosis. Here, we found that the expression of the 
310 neurotrophic factor BDNF was negatively correlated with oxidative stress and neuronal

311 apoptosis in the MCAO rats. Furthermore, previous studies have found a potential

312 protective mechanism for BDNF against mitochondrial dysfunction-related

313 neurodegenerative disorders [38]. Therefore, enhancing the secretion of BDNF

314 following I/R may represent an effective strategy for blocking the progression of strokes

315 in experimental models. Previous studies, for example, found anti-oxidative, anti-

316 apoptotic, and anti-inflammatory effects of bone marrow mononuclear cells by

317 increasing BDNF expression in the MCAO rats [39]. Similar to this, in our study, miR-

$31834 \mathrm{a}$ induced the upregulation of BDNF and demonstrated a neuroprotective effect by

319 inhibiting oxidative stress and neuronal apoptosis, which ameliorated the ischemic

320 infarction and neurological deficits. Moreover, luciferase reporter assays detected

321 BDNF as a target of miR-34a, which is consistent with our results from RIP assays [24].

322 Our findings revealed that upregulated miR-34a expression following I/R may inhibit

323 BDNF expression, resulting in oxidative stress and neuronal apoptosis. Thus, the

324 inhibition of miR-34a can upregulate BDNF expression and might be able to suppress

325 I/R injury.

326 Conclusions

327 In summary, our study demonstrated that inhibition of miR-34a blocked I/R induced 
328 injury by promoting the expression of BDNF, which may prove to be a potential and

329 promising new therapeutic target for the treatment of ischemic stroke. However, other

330 biomarkers involved in this neuronal injury that are associated with the miR-34a/BDNF

331 axis need to be further clarified for subsequent diagnosis and treatment.

\section{Acknowledgments}

333 Not applicable.

334 Data Availability

335 The data used to support the findings of this study are available from the corresponding

336 author upon request.

337 Conflicts of Interest

338 The Authors declare that there is no conflict of interest.

339 Funding Statement

340 This research did not receive any specific grant from funding agencies in the public,

341 commercial, or not-for-profit sectors. 


\section{References}

344 1. Moskowitz MA, Lo EH, Iadecola C. The science of stroke: mechanisms in search of 345 treatments. Neuron 2010;67(2):181-98.

346 2. Mozaffarian D, Benjamin EJ, Go AS, et al. Heart Disease and Stroke Statistics-2016 347 Update: A Report From the American Heart Association. Circulation 2016;133(4):e38348360.

349 3. Duan Q., Sun W., Yuan H., et al. MicroRNA-135b-5p prevents oxygen-glucose 350 deprivation and reoxygenation-induced neuronal injury through regulation of the GSK3513 beta/Nrf2/ARE signaling pathway. Arch Med Sci 2018;14(4):735-44.

352 4. Jung JE, Kim GS, Chen H, et al. Reperfusion and neurovascular dysfunction in stroke:

353 from basic mechanisms to potential strategies for neuroprotection. Molecular 354 neurobiology 2010;41:172-9.

355 5. Kaminski KA, Bonda TA, Korecki J, et al. Oxidative stress and neutrophil activation356 -the two keystones of ischemia/reperfusion injury. International journal of cardiology 357 2002;86(1):41-59.

358 6. Manzanero S, Santro T, Arumugam TV. Neuronal oxidative stress in acute ischemic 359 stroke: sources and contribution to cell injury. Neurochemistry international $360 \quad 2013 ; 62(5): 712-8$.

361 7. Ding Y., Zhu W., Kong W., et al. Edaravone attenuates neuronal apoptosis in 362 hippocampus of rat traumatic brain injury model via activation of BDNF/TrkB signaling 363 pathway. Arch Med Sci 2021;17(2):514-22.

364 8. Zhang Z., Wang B., Fei A. BDNF contributes to the skeletal muscle anti-atrophic 365 effect of exercise training through AMPK-PGC1alpha signaling in heart failure mice. 366 Arch Med Sci 2019;15(1):214-22.

367 9. Taliyan R, Ramagiri S. Delayed neuroprotection against cerebral ischemia 368 reperfusion injury: putative role of BDNF and GSK-3 $\beta$. Journal of receptor and signal 369 transduction research 2016;36(4):402-10.

370 10. Ouyang YB, Stary CM, Yang GY, et al. microRNAs: innovative targets for cerebral 371 ischemia and stroke. Current drug targets 2013;14(1):90-101.

372 11. Liu Y, Zhang X, Chen J, et al. Inhibition of mircoRNA-34a Enhances Survival of 373 Human Bone Marrow Mesenchymal Stromal/Stem Cells Under Oxidative Stress. 374 Medical science monitor : international medical journal of experimental and clinical 375 research 2018;24:264-71.

376 12. Liao L. X., Zhao M. B., Dong X., et al. TDB protects vascular endothelial cells 377 against oxygen-glucose deprivation/reperfusion-induced injury by targeting miR-34a to 378 increase Bcl-2 expression. Sci Rep 2016;6:37959. 

and Shapes Stroke Outcomes. Sci Rep 2020;10(1):3233.

381 14. Wang S. P., Wang D., Li H. X., et al. Influence of miR-34a on cerebral neuronal

382 apoptosis in rats with cerebral ischemia reperfusion through the Notch1 signaling pathway.

383 Eur Rev Med Pharmacol Sci 2019;23(18):8049-57.

384 15. Liang TY, Lou JY. Increased Expression of mir-34a-5p and Clinical Association in 385 Acute Ischemic Stroke Patients and in a Rat Model. Medical science monitor : 386 international medical journal of experimental and clinical research 2016;22:2950-5.

387 16. Chua CEL, Tang BL. miR-34a in Neurophysiology and Neuropathology. Journal of 388 molecular neuroscience: MN 2019;67(2):235-46.

389 17. Sandberg K., Umans J. G., Georgetown Consensus Conference Work Group.

390 Recommendations concerning the new U.S. National Institutes of Health initiative to

391 balance the sex of cells and animals in preclinical research. FASEB J 2015;29(5):1646$392 \quad 52$.

393 18. Liang X, Hu Q, Li B, et al. Follistatin-like 1 attenuates apoptosis via disco-interacting 394 protein 2 homolog A/Akt pathway after middle cerebral artery occlusion in rats. Stroke 395 2014;45(10):3048-54.

396 19. Liu Y., Karonen J. O., Nuutinen J., et al. Crossed cerebellar diaschisis in acute 397 ischemic stroke: a study with serial SPECT and MRI. J Cereb Blood Flow Metab 398 2007;27(10):1724-32.

399 20. Liu da Z, Jickling GC, Ander BP, et al. Elevating microRNA-122 in blood improves 400 outcomes after temporary middle cerebral artery occlusion in rats. Journal of cerebral 401 blood flow and metabolism : official journal of the International Society of Cerebral 402 Blood Flow and Metabolism 2016;36(8):1374-83.

403 21. Longa EZ, Weinstein PR, Carlson S, et al. Reversible middle cerebral artery 404 occlusion without craniectomy in rats. Stroke 1989;20(1):84-91.

405 22. Sun $H$, Zhong $D$, Wang $C$, et al. MiR-298 Exacerbates Ischemia/Reperfusion Injury 406 Following Ischemic Stroke by Targeting Act1. Cellular physiology and biochemistry: 407 international journal of experimental cellular physiology, biochemistry, and 408 pharmacology 2018;48(2):528-39.

409 23. He X, Ao Q, Wei Y, et al. Transplantation of miRNA-34a overexpressing adipose410 derived stem cell enhances rat nerve regeneration. Wound repair and regeneration: 411 official publication of the Wound Healing Society [and] the European Tissue Repair 412 Society 2016;24(3):542-50.

413 24. Cui M, Xiao H, Li Y, et al. Total abdominal irradiation exposure impairs cognitive 414 function involving miR-34a-5p/BDNF axis. Biochimica et biophysica acta Molecular 415 basis of disease 2017;1863(9):2333-41.

416 25. Guo Z., Pan R. Y., Qin X. Y. Potential Protection of Coeloglossum viride var. 
417 Bracteatum Extract against Oxidative Stress in Rat Cortical Neurons. J Anal Methods 418 Chem 2013;2013:326570.

419 26. Niizuma K., Endo H., Chan P. H. Oxidative stress and mitochondrial dysfunction as 420 determinants of ischemic neuronal death and survival. J Neurochem 2009;109 Suppl $421 \quad 1: 133-8$.

422 27. Janus K., Amelung V. E. [Integrated delivery systems in California--success and 423 failure determining factors for the first 10 years and impetus for Germany]. 424 Gesundheitswesen 2004;66(10):649-55.

425 28. Endres M., Fan G., Hirt L., et al. Ischemic brain damage in mice after selectively 426 modifying BDNF or NT4 gene expression. J Cereb Blood Flow Metab 2000;20(1):13942744.

428 29. Kleikers PW, Wingler K, Hermans JJ, et al. NADPH oxidases as a source of oxidative 429 stress and molecular target in ischemia/reperfusion injury. Journal of molecular medicine 430 (Berlin, Germany) 2012;90(12):1391-406.

431 30. Loh KP, Huang SH, De Silva R, et al. Oxidative stress: apoptosis in neuronal injury. 432 Current Alzheimer research 2006;3(4):327-37.

433 31. Dasdelen D., Solmaz M., Menevse E., et al. Increased apoptosis, tumor necrosis 434 factor-alpha, and DNA damage attenuated by 3',4'-dihydroxyflavonol in rats with brain 435 Ischemia-reperfusion. Indian J Pharmacol 2021;53(1):39-49.

436 32. Caliskan M., Mogulkoc R., Baltaci A. K., et al. The Effect of 3',4'-Dihydroxyflavonol 437 on Lipid Peroxidation in Rats with Cerebral Ischemia Reperfusion Injury. Neurochem 438 Res 2016;41(7):1732-40.

439 33. Zhao H., Tao Z., Wang R., et al. MicroRNA-23a-3p attenuates oxidative stress injury 440 in a mouse model of focal cerebral ischemia-reperfusion. Brain Res 2014;1592:65-72.

441 34. Li P, Shen M, Gao F, et al. An Antagomir to MicroRNA-106b-5p Ameliorates 442 Cerebral Ischemia and Reperfusion Injury in Rats Via Inhibiting Apoptosis and Oxidative 443 Stress. Molecular neurobiology 2017;54(4):2901-21.

444 35. Jiao D, Zhang H, Jiang Z, et al. MicroRNA-34a targets sirtuin 1 and leads to diabetes445 induced testicular apoptotic cell death. Journal of molecular medicine (Berlin, Germany) 446 2018;96(9):939-49.

447 36. Fan F, Zhuang J, Zhou P, et al. MicroRNA-34a promotes mitochondrial dysfunction448 induced apoptosis in human lens epithelial cells by targeting Notch2. Oncotarget 449 2017;8(66):110209-20.

450 37. Mattson MP, Cheng B, Smith-Swintosky VL. Mechanisms of neurotrophic factor 451 protection against calcium- and free radical-mediated excitotoxic injury: implications for 452 treating neurodegenerative disorders. Experimental neurology 1993;124(1):89-95.

453 38. Chen SD, Wu CL, Hwang WC, et al. More Insight into BDNF against 454 Neurodegeneration: Anti-Apoptosis, Anti-Oxidation, and Suppression of Autophagy. 
455 International journal of molecular sciences 2017;18(3).

456 39. Chen NN, Wang JP, Liu HF, et al. The bone marrow mononuclear cells reduce the 457 oxidative stress of cerebral infarction through PI3K/AKT/NRF2 signaling pathway. 458 European review for medical and pharmacological sciences 2017;21(24):5729-35.

459

460 

volume ( $n=5$ per group). (C) Histogram representing neurological deficit scoring $(\mathrm{n}=$ group). ${ }^{*} p<0.05$ compared with the sham group. $\# p<0.05$ compared with the MCAO group. ${ }^{*} p<0.05$ compared with the sham group. ${ }^{*} p<0.05$ compared with the MCAO group. sham group. ${ }^{\#} p<0.05$ compared with the MCAO group. 
478 representing neuronal apoptosis ( $n=5$ per group). (C) Representative images from

479 western blots for cleaved-caspase-3, Bax, and Bcl-2. (D) Histogram representing

480 western blots for cleaved-caspase-3, Bax, and Bcl-2 ( $n=5$ per group). ${ }^{*} p<0.05$

481 compared with the sham group. ${ }^{\#} p<0.05$ compared with the MCAO group. 
A

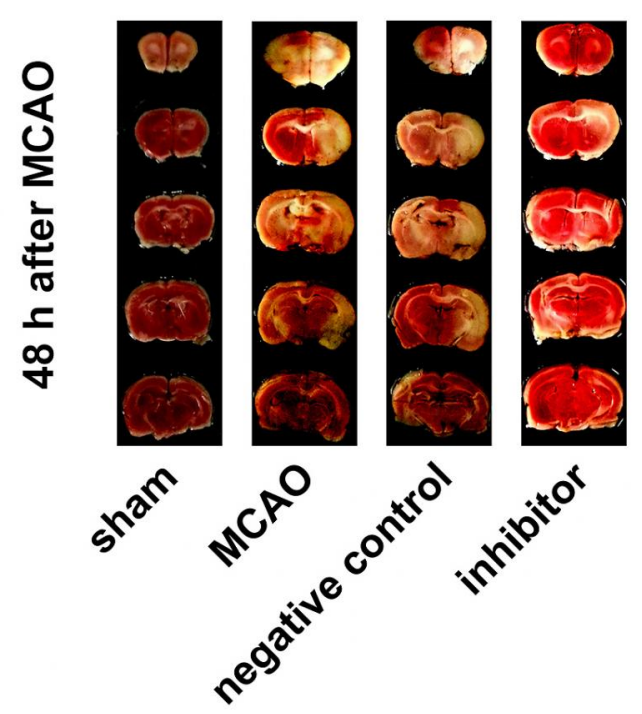

C

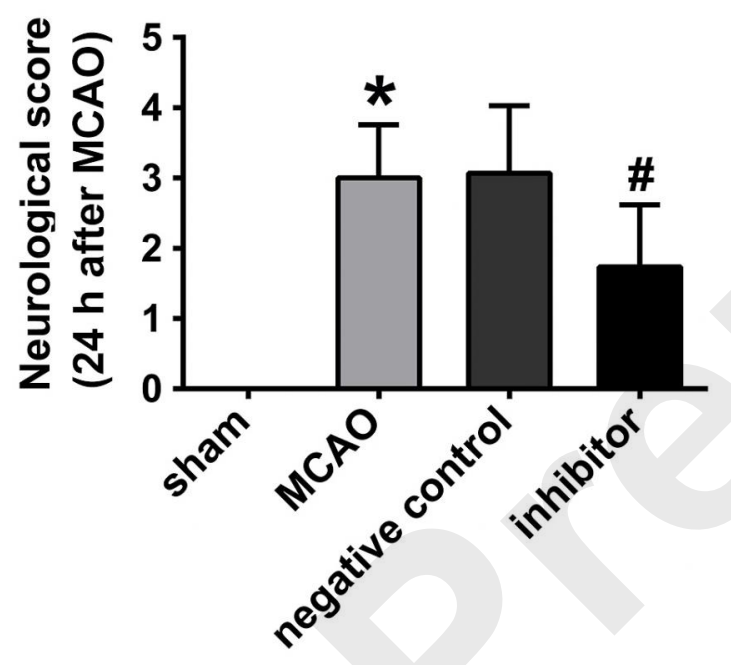

B

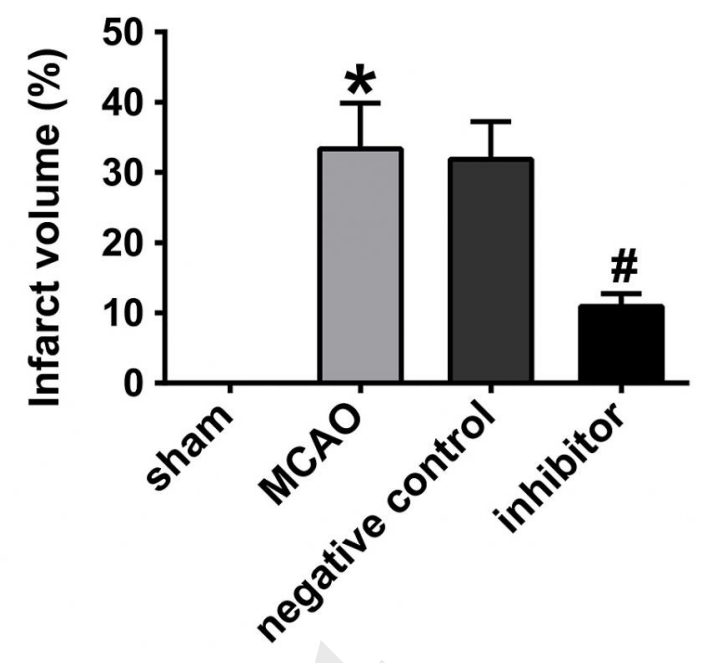

D

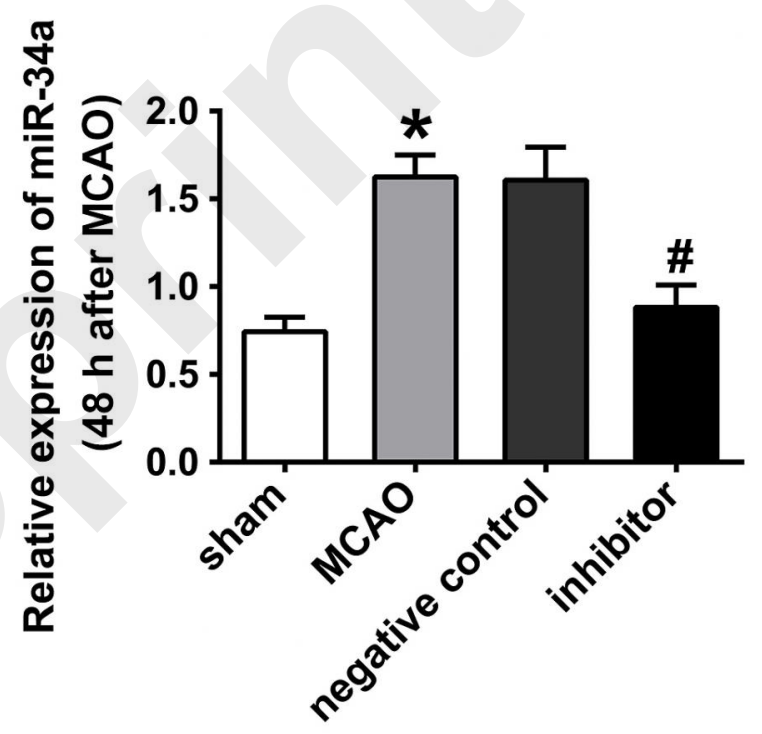

Figure 1. Inhibition of miR-34a ameliorates ischemic infarction and neurological deficits. (A) Representative images of TTC staining. (B) Histogram representing infarct volume $(n=5$ per group). (C) Histogram representing neurological deficit scoring ( $n=15$ per group). (D) Relative expression of miR-34a in the various groups ( $n=5$ per group). ${ }^{*} p<0.05$ compared with the sham group. $\# p<0.05$ compared with the MCAO group. 


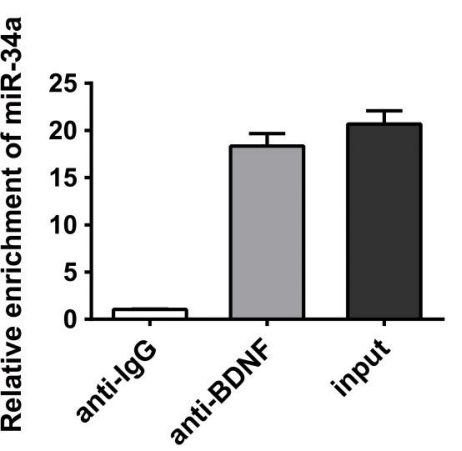

B

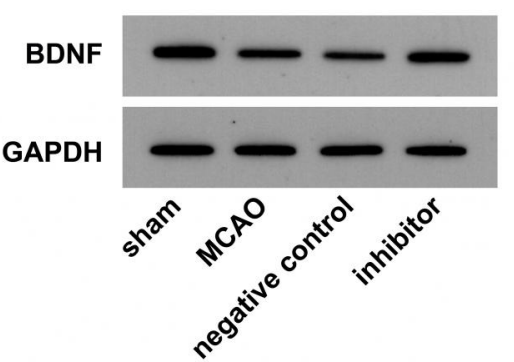

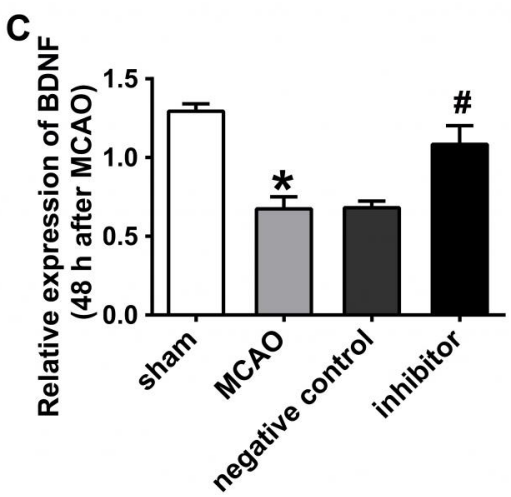

Figure 2. Inhibition of miR-34a upregulates BDNF expression. (A) Histogram representing RIP analysis ( $n=5$ per group). (B) Representative images from western blots for BDNF. (C) Histogram representing western blots for BDNF ( $n=5$ per group). ${ }^{*} p<0.05$ compared with the sham group. \#p < 0.05 compared with the MCAO group. 
A

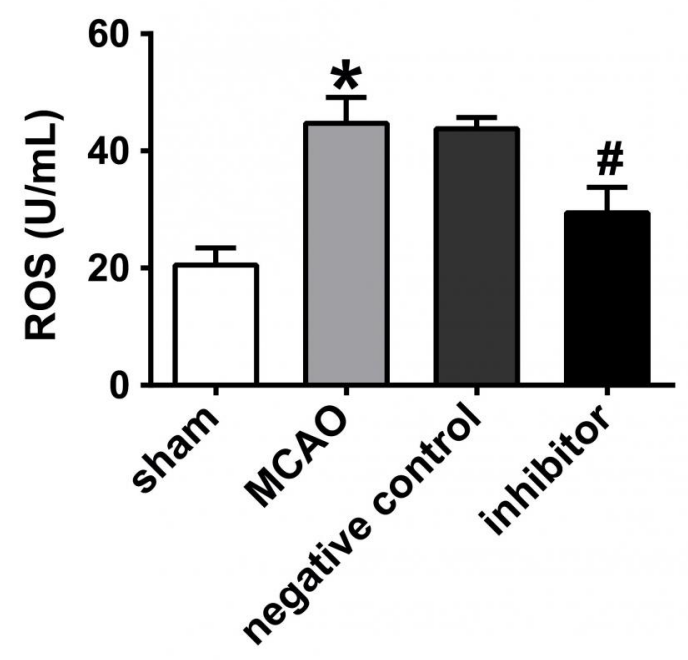

C

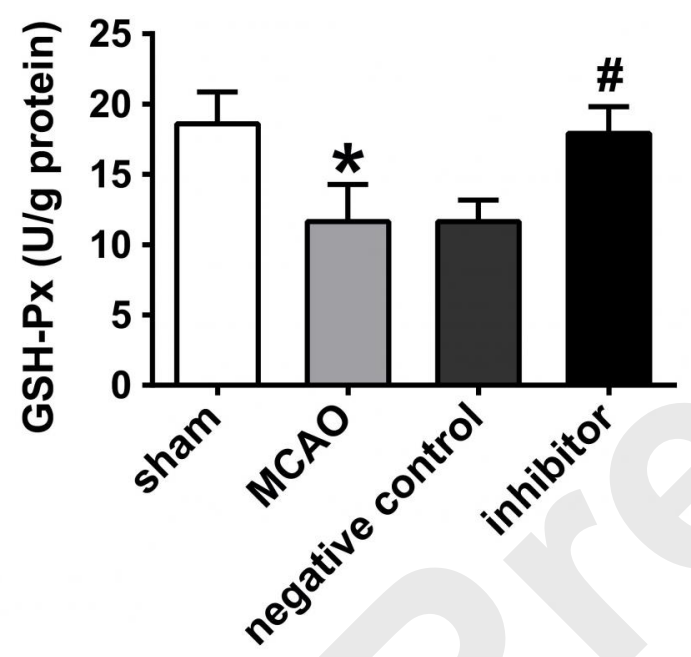

B

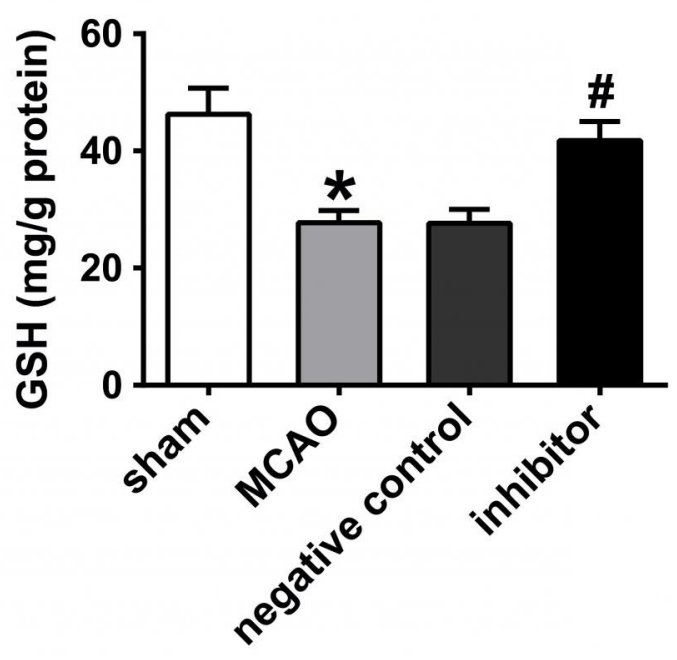

D

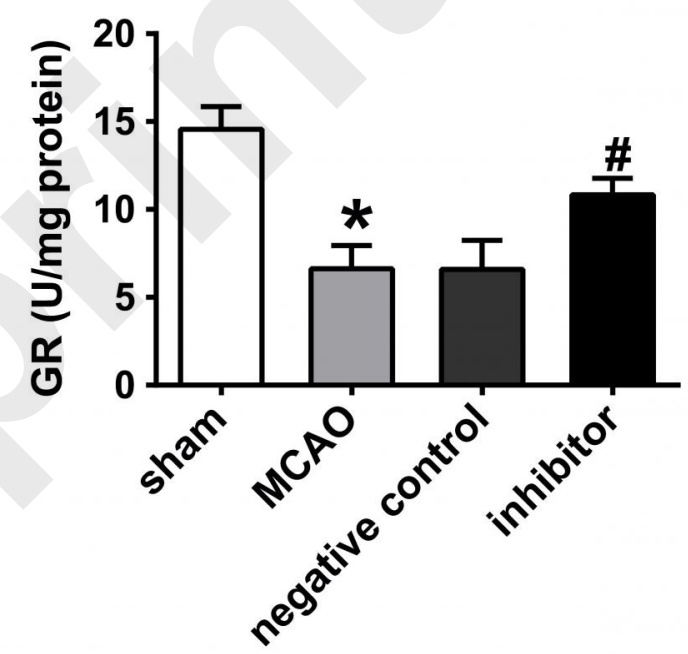

Figure 3. Inhibition of miR-34a alleviates oxidative stress following MCAO. (A) ROS concentration (arbitrary units), (B) GSH, (C) GSH-Px, and (D) GR concentrations in the various groups (arbitrary units) ( $n=5$ per group). ${ }^{*} p<0.05$ compared with the sham group. $\# p<0.05$ compared with the MCAO group. 


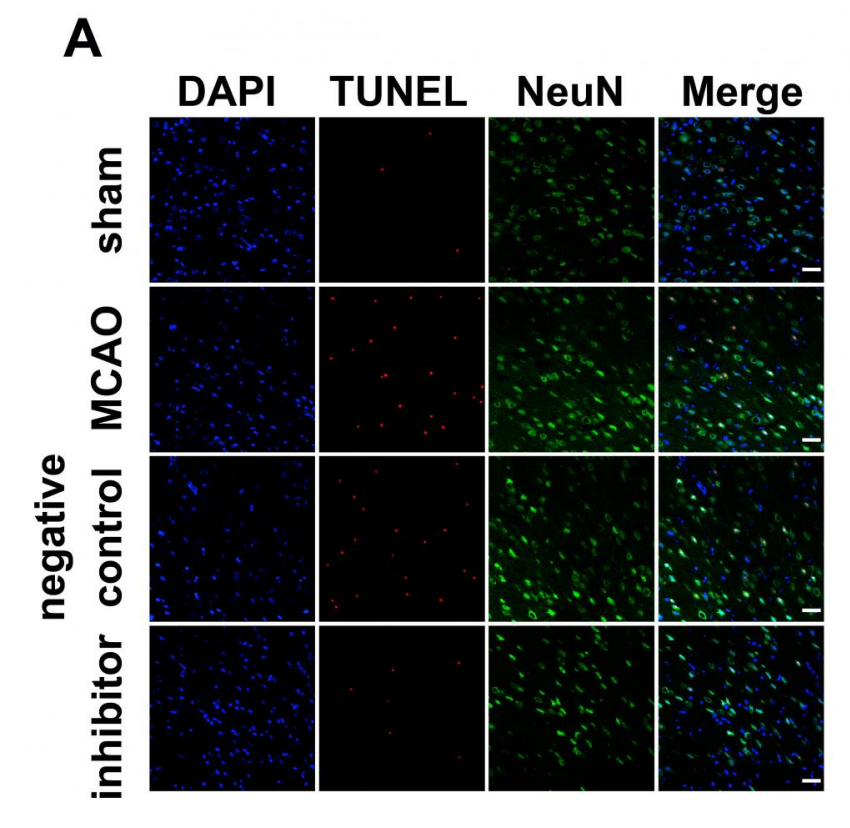

B
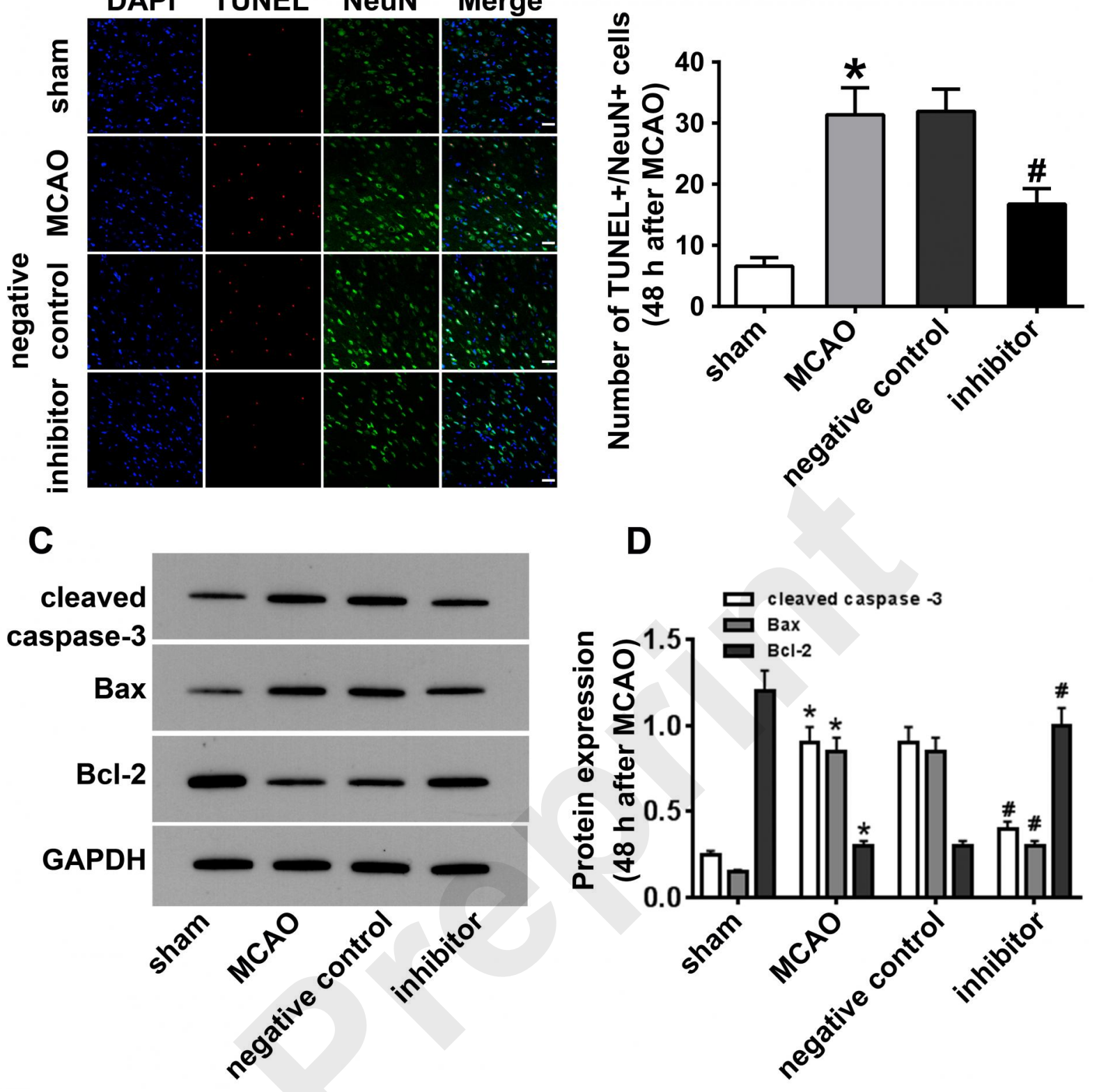

Figure 4. Inhibition of miR-34a reduces neuronal apoptosis following MCAO. (A) Representative images of TUNEL staining, scale bar: $50 \mu \mathrm{m}$. (B) Histogram representing neuronal apoptosis ( $n=5$ per group). (C) Representative images from western blots for cleaved-caspase-3, Bax, and Bcl-2. (D) Histogram representing western blots for cleavedcaspase-3, Bax, and Bcl-2 ( $n=5$ per group). ${ }^{*} p<0.05$ compared with the sham group. \#p < 0.05 compared with the MCAO group. 